\title{
Nearly Kähler submanifolds of a space form
}

\author{
Nikrooz Heidari* \\ Abbas Heydari ${ }^{\dagger}$ \\ Tarbait Modares University \\ Tarbait Modares University
}

September 25, 2018

\begin{abstract}
In this article we study isometric immersions of nearly Kähler manifolds into a space form (specially Euclidean space) and show that every nearly Kähler submanifold of a space form has an umbilic foliation whose leafs are 6-dimensional nearly Kähler manifolds. Moreover using this foliation we show that there is no non-homogeneous 6-dimensional nearly Kähler submanifold of a space form. We prove some results towards a classification of nearly Kähler hypersurfaces in standard space forms.
\end{abstract}

Keywords: Nearly Kähler manifold, Isometric immersion, Totally umbilic foliation

Mathematics Subject Classification: 53B35,53C55

\section{Introduction}

Nearly Kähler manifolds are one of the sixteen classes of almost Hermitian manifolds in the Gray-Hervella classification [12. When Gray was working in weak holonomy he faced these manifolds [10, whose Riemannian curvature operators satisfy certain identities. These identities are only slightly more complicated than and resembling the corresponding formula for Riemannian curvature operator of Kähler manifolds. Gray called them nearly Kähler manifolds and he was able to show that many results on the topology and geometry of Kähler manifolds generalize to nearly Kähler Manifolds

*nikrooz.heidari@modars.ac.ir

†aheydari@modares.ac.ir 
and discovered certain new topological and geometric properties of these manifolds 9, 11.

In 2002 Nagy [18, 19] described nearly Kähler manifolds as almost Hermitian manifolds whose canonical Hermitian connection has parallel and totally skew-symmetric torsion and showed that any complete strict nearly Kähler manifold is finitely covered by a product of homogeneous 3-symmetric manifolds, twistor spaces over quaternionic Kähler manifolds with their canonical nearly Kähler structure and 6-dimensional strict nearly Kähler manifolds.

6-dimensional nearly Kähler manifolds appearing in Nagy decomposition are Einstein of positive scalar curvature. In this dimension such a structure is characterized by the existence of some (at least locally) real Killing spinor [13. Combining this with the fact that the first Chern class vanishes one could observe that non-Kähler nearly Kähler 6-dimensional manifolds solve most of the type II string equations [8]. Other source of interest for nearly Kähler structure in six dimension is provided by their relation with geometries with torsion, $G_{2}$-holonomy and super-symmetric models.

Up to now, the only known examples of complete, 6-dimensional, strict nearly Kähler manifolds are the 6-dimensional 3-symmetric spaces endowed with their natural nearly Kähler structure, namely

$$
\begin{aligned}
S^{6} & =\frac{G_{2}}{S u(3)}, \quad S^{3} \times S^{3}=\frac{S u(2) \times S u(2)}{<1>}, \\
\mathbb{C} P^{3} & =\frac{S p(2)}{S u(2) \cdot U(1)}, \quad \mathbb{F}^{3}=\frac{S u(3)}{U(1) \times U(1)}
\end{aligned}
$$

Butruile 3] showed that these are homogeneous, complete 6-dimensional nearly Kähler manifolds. The question naturally arises that are there any nonhomogeneous complete nearly Kähler manifolds in six dimension? We have the following conjecture by Butrulle.

Conjecture 1.1. Every compact nearly Kähler manifold is a 3-symmetric space.

This conjecture is still open. By the work of Nagy[18, 19], it may be separated into two sub-conjectures. The first one is a similar conjecture on quaternionic Kähler manifolds and symmetric spaces, where there are several reasons to believe that it is true (it was solved by Poon and Salamon[20] in dimension 8 and recently by Haydee and Herrera [14] in dimension 12). The second may be formulated as: The only compact, simply connected, irreducible (with respect to the holonomy of the intrinsic Hermitian connection), 6-dimensional, nearly Kähler manifolds is the sphere $S^{6}$. This somehow concerns the core of the nearly Kähler geometry and might explain the rareness of such manifolds or the difficulty to produce non-homogeneous examples. 
In this paper, to further explore the latter conjecture, we study isometric immersions $f: M^{2 n} \longrightarrow \mathbb{Q}_{c}$ from nearly Kähler Manifolds into a space form (specially an Euclidean space) of constant curvature $c$. We show that every nearly Kähler submanifold of a space form has a foliation such the leaves of this foliation are six dimension, homogeneous nearly Kähler manifolds. As a result we show that if $n=3$ there is no non-homogeneous nearly Kähler submanifold of a space form and prove that the only 6-dimensional, irreducible (with respect to the holonomy of the intrinsic connection), complete(compact), simply connected nearly Kähler submanifolds of a space form is $S^{6}$. This proves the second part of the Batrulle conjecture in the situation that the nearly Kähler manifolds are immersed in a space form.

Theorem 1.2. Let $f: M^{2 n} \longrightarrow \mathbb{Q}_{c}^{2 n+p}$ be an isometric immersion from a complete, simply connected strictly nearly Kähler manifold into a space form of constant curvature $c$, then there is an involute totally umbilic foliation on $M$ such that the leaves of this foliation are 6-nearly Kähler homogeneous manifolds. Moreover, each leaf coincides with a 6-dimensional nearly Kähler factor appearing in the Nagy decomposition.

Corollary 1.3. Suppose that $f: M^{6} \longrightarrow \mathbb{Q}_{c}$ is an isometric immersion from a 6-dimensional complete nearly Kähler manifold into a space form, then for every $x \in M$ there is a subgroup of local isometries of $M$ which acts transitively on an open neighborhood of $x$. If $M^{6}$ is simply connected, then it is a homogeneous manifold.

Definition 1.4. An isometric immersion $f: M \longrightarrow \bar{M}$ between Riemannian manifolds called a full isometric immersion if there is no totally geodesic submanifold $N$ of $\bar{M}$ of dimension strictly less than $\operatorname{dim} \bar{M}$ such that the restriction of $f$ to $N$ is an isometric immersion.

For example $M$ is in $\mathbb{R}^{n}$ with full isometric immersion if and only if it is not contained (under $f$ ) in any affine hypersurface of $\mathbb{R}^{n}$.

Theorem 1.5. Let $f: M^{6} \longrightarrow \mathbb{R}^{n}$ be an isometric immersion from a complete (compact) simply connected strictly 6-nearly Kähler manifold into the Euclidean space. If $M$ is irreducible with respect to the holonomy of the intrinsic Hermitian connection then $M$ is locally isometric with $S^{6}$ and if $f$ is a full isometric immersion then $f$ is equivalent with natural embedding of $S^{6}$ in $R^{7}$ (namely, there exist isometric map $j: \mathbb{R}^{7} \longrightarrow \mathbb{R}^{7}$ and locally isometric map $g: M^{6} \longrightarrow S^{6}$ such that $j \circ f=i \circ g$ where $i$ is the standard embedding of $S^{6}$ as a quadric hypersurface in $\mathbb{R}^{7}$ ).

The paper is organized as follows. In section 2 basic definitions and preliminaries are given. Main reference of this section is [3]. In section 3 
we introduce complex and invariant totally umbilic foliation and prove the main theorem and its corollaries. In section 4 we use this foliation to classify nearly Kähler hypersurfaces in space forms using the principal curvature.

\section{Preliminaries}

Nearly Kähler manifolds are almost Hermitian, i.e. $2 n$-dimensional real manifolds with a $U(n)$-structure (a $U(n)$-reduction of the frame bundle) or equivalently, with a pair of tensors $(g, J)$ or $(g, \omega)$ where $g$ is a Riemannian metric, $J$ is an almost complex structure compatible with $g$ in the sense that $g(J X, J Y)=g(X, Y)$ for each $X, Y \in T M,(J$ is orthogonal with respect to $g$ point-wise) and $\omega$ is a differential 2-form, called the Kähler form, related to $g$ and $J$ by $\omega(X, Y)=g(J X, Y)$, for $X, Y \in T M$.

Associated with $g$ there is the well-known Levi-Civita connection $\nabla$ which is metric preserving and torsion-free. But nearly Kähler manifolds, as every almost Hermitian manifolds, have another natural connection $\bar{\nabla}$ called the intrinsic connection or the canonical Hermitian connection, which shall be of considerable importance in the sequel. Let $\mathfrak{s o}(M)$ be the bundle of skew-symmetric endomorphisms of the tangent space (the adjoint bundle of the metric structure). The set of metric connections of $(M, g)$ is an affine space $\mathcal{S O}$ modelled on the space of sections of $T^{*} M \otimes \mathfrak{s o}(M)$. The set $\mathcal{U}$ of Hermitian connections (connections which preserve both the metric and the almost complex structure or the Kähler form) is an affine subspace of $\mathcal{S O}$ with vector space $\Gamma\left(T^{*} M \otimes \mathfrak{u}(M)\right)$, where $\Gamma\left(T^{*} M \otimes \mathfrak{u}(M)\right)$ is the subbundle of $\mathfrak{s o}(M)$ formed by the endomorphisms which commute with $J$ (in other words, the adjoint bundle of the $U(n)$-structure). We denote the orthogonal complement of $\mathfrak{u}(M)$ in $\mathfrak{s o}(M)$ by $\mathfrak{u}(M)^{\perp}$, it is identified with the bundle of skew-symmetric endomorphisms of $T M$, anti-commuting with $J$.

Definition 2.1. The canonical Hermitian connection $\bar{\nabla}$ is the orthogonal projection of $\nabla \in \mathcal{S O}$ on $\mathcal{U}$. Equivalently, it is the unique Hermitian connection such that $\nabla-\bar{\nabla}$ is a 1 -form with values in $\mathfrak{u}(M)^{\perp}$.

The difference $\eta=\nabla-\bar{\nabla}$ is known explicitly, $\eta X=\frac{1}{2} J \circ\left(\nabla_{X} J\right)$ for all $X \in T M$, and measures the failure of the $U(n)$-structure to admit a torsionfree connection. It can be used to classify almost Hermitian manifolds. For example, Kähler manifolds are characterized by $\nabla$ being a Hermitian connection: $\bar{\nabla}=\nabla$.

Definition 2.2. Let $M$ be an almost Hermitian manifold. The following conditions are equivalent and define a nearly Kähler manifold: 
(1) the torsion of $\bar{\nabla}$ is totally skew-symmetric,

(2) $\left(\nabla_{X} J\right) X=0$ for all $X \in T M$,

(3) $\nabla_{X} \omega=\frac{1}{3} \mathfrak{i}_{X} d \omega$ for all $X \in T M$,

(4) $d \omega$ is of type $(0,3)+(3,0)$ and the Nijenhuis tensor $N$ is totally skewsymmetric.

Proposition 2.3. For a nearly Kähler manifold, the torsion of the intrinsic Hermitian connection is totally skew-symmetric and parallel, that is $\bar{\nabla} \eta=0$. Moreover, this is equivalent to $\bar{\nabla} \nabla \omega=0$ or $\bar{\nabla} d \omega=0$.

Now, suppose that the curvature of $\bar{\nabla}$ is also parallel: $\bar{\nabla} \bar{R}=0$. Then $\mathrm{M}$ is locally homogeneous or an Ambrose-Singer manifold.

Lemma 2.4. [9] Assume that $(M, g, J)$ is a nearly Kähler manifold then

$$
\begin{gathered}
\left(\nabla_{X} J\right) Y+\left(\nabla_{Y} J\right) X=0 \\
\left(\nabla_{J X} J\right) J Y=\left(\nabla_{X} J\right) J Y \\
J\left(\nabla_{X} J\right) Y=-\left(\nabla_{X} J\right) J Y=-\left(\nabla_{J X} J\right) Y \\
g\left(\nabla_{X} Y, X\right)=g\left(\nabla_{X} J Y, J X\right) \\
2 g\left(\left(\nabla_{W, X}^{2} J\right) Y, Z\right)=-\sigma_{X, Y, Z} g\left(\left(\nabla_{W} J\right) X,\left(\nabla_{Y} J\right) J Z\right)
\end{gathered}
$$

Gray used following formulas to find relation between torsion of intrinsic Hermitian connection and Riemannian curvature on nearly Kähler manifolds [11. These formulas resemble the corresponding formulas for Kähler manifolds.

$$
\begin{array}{r}
<R_{X, Y} X, Y>-<R_{X, Y} J X, J Y>=\left\|\left(\nabla_{X} J\right) Y\right\|^{2} \\
<R_{W, X} Y, Z>-<R_{W, X} J Y, J Z>=<\left(\nabla_{W} J\right) X,\left(\nabla_{Y} J\right) Z> \\
<R_{W, X} Y, Z>=<R_{J W, J X} J Y, J Z> \\
2 g\left(\left(\nabla_{W, X}^{2} J\right) Y, Z\right)=\sigma_{X, Y, Z} g\left(R_{W J X} Y, Z\right)
\end{array}
$$

It is easy to check that on every nearly Kähler manifold, the tensors $A(X, Y, Z)=<\left(\nabla_{X} J\right) Y, Z>$ and $B(X, Y, Z)=<\left(\nabla_{X} J\right) Y, J Z>$ are skewsymmetric and have type $(3,0)+(0,3)$ as (real) 3 -forms.

We need the following classical relation between the covariant derivative of the almost complex structure $J$ and its Nijenhuis tensor $N$ which is proved by a straightforward computation using

$$
4 N(X, Y)=[X, Y]+J[J X, Y]+J[X, J Y]-[J X, J Y]
$$

and the anti-symmetry of the tensors A and B defined above. 
Lemma 2.5. For every nearly Kähler manifold $(M, g, J)$ we have

$$
\forall X, Y \in \chi(M) \quad N(X, Y)=J\left(\nabla_{X} J\right) Y .
$$

In lower dimensions, the nearly Kähler manifolds are mainly determined. If $M$ is nearly Kähler with $\operatorname{dim} M \leq 4$, then $\mathrm{M}$ is Kähler. If $\operatorname{dim} M=6$, then we have the following:

Proposition 2.6. [9, 11, 24] Let $(M, g, J)$ be a 6-dimensional, strict, nearly Kähler manifold. Then

(1) $\nabla J$ has constant type, that is

$$
\left\|\left(\nabla_{X} J\right) Y\right\|^{2}=\frac{S}{30}\|X\|^{2}\|Y\|^{2}-g(X, Y)^{2}-g(J X, Y)^{2}
$$

for all vector fields $X$ and $Y$,

(2) the first Chern class of $(M, J)$ vanishes,

(3) $M$ is an Einstein manifold;

$$
\text { Ricc }=\frac{S}{6} g, \quad \text { Ricc }^{*}=\frac{S}{30} g .
$$

Moreover if the tensor $\nabla J$ has constant type $\alpha$ then $\operatorname{dim} M=6$ and $\alpha=\frac{S}{30}$ where $S$ is scalar curvature.

The next lemma follows immediately.

Lemma 2.7. For vector fields $W, X, Y$ and $Z$ we have

$$
\begin{array}{r}
g\left(\left(\nabla_{W} J\right) X,\left(\nabla_{Y} J\right) Z\right)=\frac{S}{30}\{g(W, Y) g(X, Z)-g(W, Z) g(X, Y) \\
-g(W, J Y) g(X, J Z)+g(W, J Z) g(X, J Y)\},
\end{array}
$$

and

$g\left(\left(\nabla_{W} \nabla_{Z} J\right) X, Y\right)=\frac{S}{30}\{g(W, Z) g(J X, Y)-g(W, X) g(J Z, Y)+g(W, Y)(J Z, X)\}$,

also

$$
\begin{array}{r}
\Sigma g\left(J e_{i}, e_{j}\right) R\left(e_{i}, e_{j} X, Y\right)=-\frac{S}{15} g(J x, Y), \\
\Sigma g\left(\left(\nabla_{X} J\right) e_{i}, e j\right) R\left(e_{i}, e_{j}, Y, Z\right)=-\frac{S}{30} g\left(\left(\nabla_{X} J\right) Y, Z\right),
\end{array}
$$

where $\left\{e_{i}\right\}$ is a local orthonormal frame field on $M$. 


\section{Complex and invariant totally umbilic foli- ation}

Let $f: M^{n} \longrightarrow \mathbb{Q}_{c}^{n+p}$ be an isometric immersion from a complete oriented Riemannian manifold $M$ into the space form $\mathbb{Q}$ of codimension $p$ and $\alpha$ be its second fundamental form. For smooth normal section $\eta \in \Gamma(T M)^{\perp}$ and $x \in M$ the totally umbilic distribution (with singularity) associated to $\eta$ is defined by

$$
\Delta_{x}=\left\{X \in T_{x} M \mid \alpha(X, Y)=<X, Y>\eta \quad \forall Y \in T_{x} M\right\} .
$$

This distribution is smooth because $\Delta_{x}=\bigcap \operatorname{Ker} \alpha_{i}(x)$ where

$$
\alpha_{i}=\alpha\left(\cdot, X_{i}\right)-<\cdot, X_{i}>\eta
$$

is a smooth 1-form for the local frame fields $\left\{X_{i}\right\}$. Therefore $\Delta$ is a smooth distribution with singularity (may not be of constant dimension) because these 1-forms may be linearly dependent. Put $\nu(x)=\operatorname{dim} \Delta_{x}$, then $\nu$ is semi-continuous and there is exist an open $U$ such that $\nu$ is constant on $U$. This distribution is involutive because by the Codazzi equation we have

$$
\left(\nabla_{X}^{\perp} \alpha\right)(Y, Z)=\left(\nabla_{Y}^{\perp} \alpha\right)(X, Z)
$$

hence

$\nabla_{X}^{\perp} \alpha(Y, Z)-\alpha\left(\nabla_{X} Y, Z\right)-\alpha\left(Y, \nabla_{X} Z\right)=\nabla_{Y}^{\perp} \alpha(X, Z)-\alpha\left(\nabla_{Y} X, Z\right)-\alpha\left(X, \nabla_{Y} Z\right)$

and if $X, Y \in \Delta_{x}$ then for all $Z \in \Delta_{x}^{\perp}$ we have

$$
\alpha\left(\nabla_{X} Y, Z\right)+<\nabla_{X} Y, Z>\eta=\alpha\left(\nabla_{Y} X, Z\right)+<\nabla_{Y} X, Z>\eta
$$

and for $Z \in \Delta_{x}$ there is nothing to prove. On the other hand, $[X, Y]=$ $\nabla_{X} Y-\nabla_{Y} X$, therefore

$$
\alpha([X, Y], Z)=<[X, Y], Z>\eta
$$

Put

$$
\Delta_{x}^{\prime}=\left\{X \in T_{x} M \mid \forall Y \in T_{x} M \quad \alpha(X, J Y)+\alpha(J X, Y)=0\right\},
$$

where $J$ is the almost complex structure. Like the previous case, this distribution is smooth but may not be involutive. When $M$ is almost Hermitian, 
the complexification of totally umbilic distribution at each point is defined by

$$
\Delta_{x} \cap J \Delta x=\Delta_{x} \cap \Delta_{x}^{\prime}
$$

This distribution is smooth but may be not involutive. When $M$ is nearly Kähler with torsion $T$ of intrinsic Hermitian connection, we define

$$
\Delta_{x}^{\prime \prime}=\left\{X \in T_{x} M \mid \forall Y, Z \in T_{x} M \quad \alpha(T(X, Y), Z)+\alpha(T(X, Z), Y)=0\right\} .
$$

The corresponding distribution is smooth but not involutive.

Definition 3.1. Let $f: M^{2 n} \longrightarrow \mathbb{Q}^{2 n+p}$ be an isometric immersion from a complete nearly Kähler manifold $M$ into a space form $\mathbb{Q}$. We denote by $\alpha$ the second fundamental form of $f$ and by $T$ the torsion of intrinsic Hermitian connection on $M$ and define the complex and invariant totally umbilic distribution at each point by

$$
D_{x}^{\eta}=D_{x}=\Delta_{x} \cap \Delta_{x}^{\prime} \cap \Delta_{x}^{\prime \prime}
$$

which can be easily seen to be equal to

$$
D_{x}=\left\{X \in T_{x} M \mid \forall Y \in T_{x} M \quad X \in \Delta_{x}, J X \in \Delta_{x}, T(X, Y) \in \Delta_{x}\right\}
$$

Lemma 3.2. Let $f: M^{2 n} \longrightarrow \mathbb{Q}_{c}^{2 n+p}$ be an isometric immersion from a complete and strictly nearly Kähler manifold into a space form of curvature $c$ and codimension $p$. Then the complex and invariant totally umbilic distribution is smooth and involutive and it defines a foliation with singularity. The leaf of this foliation may not be complete even if $M$ is compete.

Proof. By definition and smoothness of $\Delta_{x}, \Delta_{x}^{\prime}, \Delta_{x}^{\prime \prime}$ we conclude that $D_{x}$ is smooth. Therefore $D_{x}$ defines a singular distribution on $M$. We show that this distribution is involutive. Let $X, Y \in D_{x}$ by Lemma 2.5 and the fact that $D_{x}$ is invariant under $T, J$ in $\Delta$ we have $N(X, Y) \in D_{x}$ where $N$ is the Nijenhuis tensor of almost complex structure $J$. By definition of $N$ and since $\Delta_{x}$ is involutive we have

$$
N(X, Y)-[X, Y]+[J X, J Y]=J[J X, Y]+J[X, J Y] \in \Delta_{x} .
$$

For each $Z \in \Delta_{x}^{\perp}$ we have $<J[X, Y],-J[J X, J Y], Z>=0$, therefore

$$
\alpha(J[X, Y], Z)=\alpha(J[J X, J Y], Z),
$$


and a computation like that of [6, 7] and using fact that $N$ is of skewsymmetric implies that $J[X, Y] \in \Delta_{x}$.

To show that the distribution $D$ is involutive we show that for all $Z \in T_{x} M$, $T([X, Y], Z) \in \Delta_{x}$. For this purpose we define the following tensor

$$
\begin{aligned}
& C_{X}: D_{x}^{\perp} \longrightarrow D_{x}^{\perp} \\
& C_{X}(Z)=T(X, Z)^{\perp} .
\end{aligned}
$$

Lemma 3.3. The tensor $C_{X}$ vanishes for all $X \in D_{x}$.

Proof. Let $Y \in D_{x}$ be such that $\langle X, Y\rangle=1$ and $W \in \Delta_{x}^{\perp}$. Note that $T$ is $\bar{\nabla}$-parallel and we have

$$
0=\left(\nabla_{X} T\right)(Y, Z)+T(X, T(Y, Z)-T(T(X, Y), Z)-T(Y, T(X, Z)),
$$

and since $X, Y \in D_{x},<T(X, T(Y, Z), W>=<T(Y, T(X, Z), W>=0$, thus using Gray formula at the end of Lemma 2.4 we conclude that

$$
\begin{aligned}
<T(T(X, Y), Z), W> & =<T(X, Y), T(W, Z)>=<R_{X, J Y} J W, Z> \\
& +<R_{X, J Y} W, J Z>
\end{aligned}
$$

and if we use described Riemannian curvature by the second fundamental form

$$
\begin{aligned}
<R_{X, Y} Z, W> & =c\{<X, W><Y, Z>-<X, Z><Y, W>\} \\
& +<\alpha(X, W), \alpha(Y, Z)>-<\alpha(X, Z), \alpha(Y, W)>
\end{aligned}
$$

we get $\left\langle T(T(X, Y), Z, W\rangle=0\right.$ (because $\left.X, Y \in D_{x}\right)$. Therefore by Gray formula (8) we have

$$
\begin{aligned}
& 0=\sigma_{Y, Z . W}<R_{X, J Y} Z, W>=<\alpha(X, Y), \alpha(J Z, W)-\alpha(Z, J W)> \\
& \Longrightarrow<\eta, \alpha(J Z, W)-\alpha(Z, J W)>=0 .
\end{aligned}
$$

Remark 3.4. Note that for $A \in T_{x} M, X \in D_{x}, Z \in D_{x}^{\perp}$ and $W \in \Delta_{x}^{\perp}$,

$$
<\left(\nabla_{A} T\right)(X, Z), W>=<\left(\nabla_{A, X}^{2} J\right) J Z, W>,
$$

Now to show that the tensor $C$ vanishes, let $A \in T_{x} M$, it is sufficient to show that for all $W \in \Delta_{x}^{\perp},<T(T(X, Z), A), W>$ vanishes. But

$$
\begin{aligned}
<T(T(X, Z), A), W> & =<\left(\nabla_{A} T\right)(Z, X), W>+<T(X, T(A, Z)), W> \\
& +<T(T(A, X), Z), W>
\end{aligned}
$$


and similar to the previous case, the last two terms on the right hand side are zero. Therefore

$$
\begin{aligned}
& <T(T(X, Z), A), W>=<\left(\nabla_{A} T\right)(X, Z), W>=\frac{1}{2} \sigma_{X, Z, W}<R_{A, J X} Z . W> \\
& =<\alpha(A, W), \alpha(J X, Z)>+<\alpha(A, Z), \alpha(J X, W)> \\
& +\frac{1}{2}<\alpha(A, X), \alpha(J Z, W)-\alpha(J W, Z)>
\end{aligned}
$$

when $J X \in D_{x}, Z \in D_{x}^{\perp}$ and $W \in \Delta_{x}^{\perp}$ the first two terms on the right hand side are also zero because $X \in D_{x}$ and $\alpha(X, A)=<X, A>\eta$ therefore the third term in the above equation vanishes. This means that $T(T(X, Z), A) \in$ $\Delta_{x}$ for all $A \in T_{x} M$, therefore $T(X, Z) \in D_{x}$ and the proof is complete.

To proof that the distribution is involutive it is sufficient to show that for all $X, Y \in D_{x}$ and $Z \in T_{x} M, T([X, Y], Z) \in \Delta_{x}$. The case $Z \in D_{x}$ is trivial therefore we may suppose that $Z \in D_{x}^{\perp}$. Also we may assume that $W \in \Delta_{x}^{\perp}$ then

$$
0=<\bar{\nabla}_{X} T(Y, Z), W>=<\left(\nabla_{X} T\right)(X, Y)>+<T(X, T(Y, Z)), W>,
$$

where the last term vanishes since $X \in D_{x}$ and $T(X, T(Y, Z)) \in \Delta_{x}$ is equal to zero therefore

$$
0=<\nabla_{X} T(Y, Z), W>-<T\left(\nabla_{X} Y, Z\right), W>-<T\left(Y, \nabla_{X} Z\right), W>
$$

the last term of this relation is also zero since the $Y \in D_{x}$ and

$$
\begin{aligned}
<T\left(\nabla_{X} Y, Z\right), W> & =<\nabla_{X} T(Y, Z), W>=<T(Y, Z), \nabla_{X} W> \\
& =-<T\left(Y, \nabla_{X} W\right), Z>=0 .
\end{aligned}
$$

The last term is equal zero by Lemma 3.3 therefore distribution is involutive. We know that the dimension of $\Delta$ on integral curves associated with the vector fields generated this distribution is constant [4]. With a similar argument and using [22] we observe that $D$ is constant on integral curves associated with the generating vector fields of $D$ therefore by Sussman-Stefan theorem [17, 23] $D$ is a foliation with singularity and has maximal locally integral manifolds.

Lemma 3.5. Let $f: M^{2 n} \longrightarrow \mathbb{Q}_{c}^{2 n+p}$ be an isometric immersion from a complete and strictly nearly Kähler manifold into a space form with curvature $c$ and codimension $p$. Each leafs of complex and invariant totally umbilic foliation by torsion of the intrinsic Hermitian connection is 6-dimensional homogeneous nearly Kähler manifolds. 
Proof. Let $\theta(x)=\operatorname{dim} D_{x}$ then by Lemma 3.2 and Sussman-Stefan theorem concluded that semi-continuous function $\theta(x)$ is locally constant. It means that for all $x \in M$ there exists an open subset $U \subseteq M$ containing $x$ such that $\theta(x)$ on $U$ is constant therefore $D_{x}$ on $U$ is integrable distribution. The maximal integral manifold $N$ of this distribution is nearly Käler manifold with induced almost complex structure and inherited metric because $D$ is invariant under $J, T$. We assert that $N$ has constant type $c+<\eta, \eta\rangle$. By the Gray formula 8 in Lemma 2.4 for all $X, Y \in \chi(N)$

$$
\begin{aligned}
\left\|\left(\nabla_{X} J\right) Y\right\|^{2}= & -<R_{X, Y} X, Y>+<R_{X, Y} J X, J Y> \\
& =<\alpha(X, Y), \alpha((Y, X)>-<\alpha(X, X), \alpha(Y, Y)> \\
& -<\alpha(X, J Y), \alpha(Y, J X)>+<\alpha(X, J X), \alpha(Y, J Y) \\
& =\|\eta\|^{2}\left(-<X, Y>^{2}+<X, X><Y, Y>\right. \\
& +<X, J Y><Y, J X>) \\
& =\|\eta\|^{2}\left(\|X\|^{2}\|Y\|^{2}-<X, Y>^{2}-<X, Y>^{2}\right),
\end{aligned}
$$

hence by Proposition 2.6 $N$ is 6-dimensional manifold and $\|\eta\|^{2}=\frac{S}{30}$ where $S$ be scalar curvature of $N$. Also because $N$ is Einstein $\eta$ is of constant length. By the definition of the tangent bundle $T N$ at each point we have $H=\eta$ where $H$ is mean curvature vector field of $N$ as a submanifold of $\mathbb{Q}$.

Noted that the tangent space of this leaf at each point $x$ is $D_{x}$ therefore

$$
\begin{aligned}
<R\left(X_{i}, X_{j}\right) X_{k}, X_{l}> & =<\alpha\left(X_{i}, X_{l}\right), \alpha\left(X_{j}, X_{k}\right)>-<\alpha\left(X_{i}, X_{k}\right), \alpha\left(X_{j}, X_{l}\right)> \\
& =\|\eta\|^{2}\left\{\delta_{l}^{i} \delta_{k}^{j}-\delta_{k}^{i} \delta_{l}^{j}\right\}
\end{aligned}
$$

and a computation like that of [15] implies that $\bar{\nabla} \bar{R}=0$. Therefore by the above remark each leaf is an Ambrose-Singer manifold (locally homogeneous).

Since the Nagy decomposition to homogeneous 3-symmetric nearly Kähler, twistor space on positive Kähler quaternion and 6-dimension nearly Kähler manifold factors are unique up to homothety the proof of theorem 1.2 is complete.

Proposition 3.6. In Nagy decomposition, we have 6-dimensional factor if and only if there exist $0 \neq \eta \in T_{f}^{\perp} M$ such that the $D^{\eta}$ is non-zero distribution.

Proof. By Theorem 1.2 the leaves of foliation $D$ are isometric to 6-dimensional factor in Nagy decomposition.

Conversely, let $N$ be the 6-dimensional nearly Kähler factor in Nagy decomposition with second fundamental form $\beta$ as a submanifold of $M$, so 
$\beta(X, J Y)=J \beta(X, Y)$ for all $X, Y \in T N$. One can consider $N$ as a submanifold of $\mathbb{Q}^{2 n+p}$ (by $N^{6} \longrightarrow M^{2 n} \longrightarrow \mathbb{Q}^{2 n+p}$ ). If $H$ is the mean curvature of $N$ in $\mathbb{Q}$ we choose $\eta$ parallel to $H=\sum_{i=1}^{i=3} \beta\left(e_{i}, e_{i}\right)+\beta\left(J e_{i}, J e_{i}\right)$ such that $c+\|\eta\|=\frac{S}{30}$, where $\left\{e_{i}, J e_{i}\right\}$ is suitable Watanabe frame [24] for $N$ and $S$ is the scalar curvature of $N$. Therefore $\eta \in T_{f}^{\perp} M$ and $T^{M}(X, Y)=T^{N}(X, Y)$ for all $X, Y \in T N$ where $T^{N}$ and $T^{M}$ are torsions of canonical Hermitian connection on $N$ and $M$ respectively. By computation like as the proof of lemma 3.5 we have $\beta(X, Y)=g(X, Y) \eta$ for all $X, Y \in T N$. So $D^{\eta}$ is non-zero.

Proof. (corollary 1.3) From the previous lemma, if $M$ is 6-dimensional each leaf of complex and invariant totally umbilic foliation is an open subset of $M$ and because each leaf is locally homogeneous, $M$ is locally homogeneous and by Ambrose-Singer theorem if $M$ is simply connected then $M$ is homogeneous. Therefore there is no non-homogeneous complete simply connected and strict nearly Kähler submanifold of a space form, namely such a manifold must be one in the Butrulle classification of complete homogeneous 6-nearly Kähler manifolds.

Proof. (theorem 1.5) Since $M$ has irreducible holonomy of intrinsic Hermitian connection, from the proof of Nagy decomposition each leaf of complex and invariant totally umbilic foliation is complete. Therefore by Corollary 1.3 each leaf of this foliation on $M$ is open, compact and thus is closed therefore as $M$ is connected, the only leaf is itself $M$. But $M$ is simply connected hence it must be one of the manifolds listed in Butrulle theorem. Also from [1] we know that $\mathbb{C} P^{3}, \mathbb{F}^{3}$ with their standard nearly Kähler structure coming from the twistor construction have reducible holonomy of intrinsic Hermitian connection and $S^{3} \times S^{3}$ is Riemannian reducible. Therefore the only manifold which is irreducible with respect to the holonomy of the intrinsic Hermitian connection must be $S^{6}$.

Remark 3.7. The only totally umbilic irreducible Euclidean submanifold is the sphere and the only sphere with strictly nearly Kähler structure is 6sphere.

If $\gamma$ is a normal vector field on $M$ which does not vanish everywhere, for the complex and invariant totally umbilic foliation $D^{\gamma}$ we have $T_{x} M=D_{x}^{\gamma}=$ $D_{x}^{\eta}$ and for $0 \neq X \in T_{x} M, \alpha(X, X)=<X, X>\eta_{x}=<X, X>\gamma_{x}$ thus $\eta_{x}=\gamma_{x}$ for all $x \in M$, therefore

$$
\mathcal{N}_{x}^{1}:=\operatorname{spam}\left\{\alpha(X, Y) \mid X, Y \in T_{x} M\right\}=<\eta_{x}>.
$$

In particular, $\mathcal{N}^{1}$ is an invariant distribution under parallel translation with respect to the induced connection on normal bundle $\nabla^{\perp}$, thus by reduction 
of codimension theorem [5] there exist a $(6+1)$-dimensional totally geodesic submanifold $N$ of $\mathbb{R}^{n}$ such that $f$ is an isometric immersion of $M$ into $N$. This contradicts the fact that $f$ is full isometric immersion, therefore $n$ must be 7 . The rest part of theorem is already considered in [2].

\section{Classification of nearly Kähler hypersur- faces of a space form}

In this section using complex and invariant totally umbilic foliations we classify and describe nearly Kähler hypersurfaces of a space form based on their principal curvatures.

In the previous section, we observed that there is no non-homogeneous complete nearly Kähler submanifold in a space form. If $\operatorname{dim} M=6$ and $M$ is simply connected, there exist only four cases (up to homothety): $S^{6}, S^{3} \times S^{3}$, $\mathbb{C} P^{3}$ and $\mathbb{F}^{3}$.

Using Reyes Carrion structure theorem [21] and Theorem1.1 of [1] which state that every complete Nearly Kähler, non-Kähler manifold, whose canonical connection has reduce holonomy is homothetic to $\mathbb{C} P^{3}$ or $\mathbb{F}^{3}$ with their standard nearly Kähler structure, we show that these type of manifolds cannot be isometrically immersed hypersurfaces in a standard space form.

Theorem 4.1. If $f: M^{6} \longrightarrow \mathbb{Q}_{c}^{7}$ is an isometric immersion from a complete, simply connected and 6-dimensional nearly Kähler manifold into a standard space form with curvature $c$ then $M$ is a homogeneous Riemannian manifold and one of the following holds (up to homothty):

(i) $M$ is $S^{6}$, the space form is $\mathbb{R}^{7}$ and $f$ is equivalent to the standard embedding.

(ii) $M$ is $S^{3} \times S^{3}$, the space form is 7-sphere with $c=\sqrt{2}$ and $f$ is equivalent to the standard embedding $S^{3} \times S^{3}$ in $S_{\sqrt{2}}^{7}$.

Proof. : Let $M$ be a 6-dimensional nearly Kähler manifold such that the holonomy group of the intrinsic Hermitian connection $\bar{\nabla}$ is strictly contained in $S U_{3}$. As every maximal subgroup of $S U_{3}$ is conjugate to $U_{2}$, this is equivalent to the reducibility of $\mathrm{M}$. We fix such a $U_{2}$ containing the holonomy group, which defines a $\bar{\nabla}$-parallel complex line sub-bundle of $T M$, henceforth denoted by $\nu$. Since $\nabla_{X} Y=\bar{\nabla}_{X} Y$ where $X$ and $Y$ are complex linearly dependent, we deduce that $\nu$ is totally geodesic for $\nabla$ (and in particular integrable). The orthogonal complement of $\nu$ in $T M$ will be denoted by $\mathcal{H}$ and the restriction of $\mathcal{H}$ to every integral manifold $S$ of $\nu$ is identified with 
the normal bundle of $S[1$. Therefore there exists a Riemannian manifold $N$ and a Riemannian submersion with totally geodesic fibers $\pi: M \longrightarrow N$ such that the tangent space of the fiber through any point $x \in M$ is $\nu_{x}$ [1]. Moreover, $N$ is an oriented self-dual, Einstein with positive scalar curvature and $\pi: M \longrightarrow N$ is the $S^{2}$-bundle over $N$ whose fiber over $x \in N$ consists of all complex structures on $T_{x} N$ that are compatible with the metric and orientation[21]. We show that $M$ could not be a hypersurface in space form. For every $x \in N$ there exist open simply connected neighborhood $U$ of $x$ such that $\pi^{-1}(U) \approx U \times S^{2}$. Indeed this is an isometry because by pulling back the metric of $U \times S^{2}$ on $\pi^{-1}(U)$ we have a 6-dimensional nearly Kähler manifold which is locally homogeneous by theorem 1.2 and corollary 1.3. By Butrulle theorem [3] this metric coincides with the metric inherited from $M$. Now, if $M$ is a hypersurface in the standard space form, restricting the isometric immersion from $M$ into $\mathbb{Q}^{7}$ to $\pi^{-1}(U)$ and composing it with the isometry coming from $\pi^{-1}(U) \approx U \times S^{2}$ there exist an isometric immersion from $U \times S^{2}$ into $\mathbb{Q}^{7}$. If the space form is the Euclidean space then we have a contradiction with the fact that $N$ has positive scalar curvature. If the space form is a sphere then again we get a contradiction with the fact that $N$ is self-dual or non-compact. Therefore complete simply connected nearly Kähler hypersurface of a space form could not have reduce holonomy of intrinsic Hermitian connection and holonomy of intrinsic Hermitian connection must be $S U_{3}$, by the main theorem of [1] this hypersurface can not be neither $\mathbb{C} P^{3}$ nor $\mathbb{F}^{3}$. By Theorem 1.2 this hypersurface is homogeneous and must be one of $S^{6}$ or $S^{3} \times S^{3}$ and the result follows from corollary 1.3 .

For a $2 n$-dimensional nearly Kähler manifold $M$ with strictly positive Ricci curvature if $M$ is a hyperspace in Euclidean space then $\operatorname{Ric}\left(X_{i}, X_{i}\right)=$ $\lambda_{i} \sum_{j \neq i} \lambda_{j}>0$ where $\lambda_{i}$ 's are principal curvatures and $X_{i}$ 's are the corresponding eigenvectors. In particular $\lambda_{i}$ 's are all positive or all negative. Thus the Gaussian curvature of the hypersurface, $\lambda_{1} \ldots \lambda_{2 n}$, is strictly positive. Hence if $M$ is complete, the Guassian sphere map $M \longrightarrow S^{2 n}$ is a diffeomorphism [16]. In particular $M$ is a strictly convex hypersurface.

If $M$ is connected and simply connected the leaves of complex and invariant intrinsic Hermitian totally umbilic foliation on $M$ are homogeneous 6-nearly Kähler manifolds by our main theorem. When $M$ is a hypersurface in the Euclidean space all the six number of principle curvatures coincide.

When $n=4$, the remaining principle curvatures $\lambda_{7}, \lambda_{8}$ are not equal. Otherwise, if since the distribution $x \longmapsto\left\{X \in T_{x} M \mid A X=\lambda_{7} X\right\}$ is invariant under almost complex structure and torsion of intrinsic Hermitian connec- 
tion (because $X_{7}=J X_{8}$ ) each leaf of this foliation must have dimension six which is impossible. Therefore every complete simply connected strictly 8-nearly Kähler hypersurface in the Euclidean space must be isometric with a product of a 6-dimensional nearly Kähler hypersurface in the Euclidean space (listed above theorem) with a compact, oriented 2-dimensional surface with positive Guassain curvature and non-equal principal curvatures.

For $n=5$ by similar argument one cane show that four remaining principle curvatures are mutually distinct.

\section{References}

[1] F. Belgun and A. Moroianu, Nearly Kähler 6-manifolds with reduce holonomy, Ann. Global Anal. Geom. 19 (2001), 307-319.

[2] J. Berndt and S. Console and C. Olmose, Submanifolds and Holonomy, Research Notes in Mathematics Series, Champman\& Hall/CRC, 2003.

[3] J.-B. Butruille, Homogenous nearly Kähler manifolds, in: Handbook of pseudo-Riemannian geometry and supersymmetry, Vicente Corts Ed., vol. 16, IRMA Lect. Math. theor. Phys Eur. Math. Soc. Publishing House, Zurich, 2010, 399-423.

[4] M. Dajczer, Submanifolds and isometric immersions, Mathematics Lecture Series, Publish or Perish, 1990.

[5] J. Erbacher, Reduction of the codimension of an isometric immersion, J. Differential Geometry 5, (1971), 333-340.

[6] A. Florit and F. Zheng, A local and global splitting result for real Kähler Euclidean submanifolds, Arch. Math. 84 (2005) 88-95.

[7] A. Florit and F. Zheng, Complete real Kähler submanifolds in codimension two, Math. Z. 258 (2008) 291-299.

[8] T. Friedrich, Nearly Kähler and nearly parallel $G_{2}$-structures on spheres, Arch. Math. 42 (2006), no.5, 241-243.

[9] A. Gray, Nearly Kähler Manifolds, J. Differential Geometry 4 (1970), 283-309.

[10] A. Gray, Weak holonomy groups, Math. Z. 123 (1971), 290-300.

[11] A. Gray, The structure Of nearly Kähler Manifolds, Math. Ann., 223 (1976), 233-248. 
[12] A. Gray and M. Hervella, The sixteen classes of almost Hermitian manifolds and their linear invariants, Ann. Mat. Pura Appl. 123 (1980), no.1, $35-58$.

[13] R. Grunewland, Six-dimensional Riemannain manifolds with a real Killing spinor, Ann. Global Anal. Geom. 8 (1990), no.1, 43-59.

[14] H. Haydeé and R. Herrera, Â-genus on non-spin manifolds with $S^{1}$ actions and the classification of positive quaternion-Kähler 12-manifolds, J. Differential Geom. 61, (2002), 341-364.

[15] N. Heidari and A. Heydari, 6-Nearly Kähler hypersurfaces in space forms, in: Proceeding of Conference (the 43rd Annual Iranian Mathematics Conference),27-30 August 2012, University of Tabriz, 394-397.

[16] S. Kobayashi and K. Nomizu, Foundations of differential geometry, Vol. I,II, Wiley, 1963, 1969.

[17] P.-W. Michor, Topics in differential geometry, Amer. Math. Soc., 2008.

[18] P.-A. Nagy, Nearly Kähler geometry and Riemannain foliations, Asian J. Math. 6 (2002), no.3, 481-504.

[19] P.-A. Nagy, On Nearly-Kähler geometry, Ann. Global Anal. Geom., 22 (2002), 167-178.

[20] Y.-S. Poon and S.-M. Salamon, Quaternionic Kähler 8-manifolds with positive scalar curvature, J. Differential Geom. 33, (1991), 363-378.

[21] R. Reys-Carrion, Some special geometry defined by Lie groups, $\mathrm{PhD}$ thesis, Oxfords, 1993.

[22] P. Stefan, Accessibility and foliations with singularities, Bull. Amer. Math. Soc. 80, (1974), no.6, 1142-1145.

[23] H.-J. Susmman, Orbits of families of vector fields and integrablity of systems with sigularities, Bull. Amer. Math. Soc. 79, (1973), no.1, 197199.

[24] Y. Watanabe and Y. Jinsuh, On 6-dimensional nearly Kähler manifolds, Canad. Math. Bull. 53 (2010), no.3, 564-570. 